This article has been accepted for publication in Decision Support Systems. Please refer to

this article as "Defraeye, M., Van Nieuwenhuyse, I. (2012). Controlling excessive waiting

times in small service systems with time-varying demand: an extension of the ISA algorithm.

Decision Support Systems, Available online 6 June 2012, ISSN 0167-9236,

DOI: 10.1016/j.dss.2012.05.058. (http://www.sciencedirect.com/science/article/pii/

S0167923612001790)

\title{
Controlling excessive waiting times in small service systems with time-varying demand: an extension of the ISA algorithm
}

Mieke Defraeye $^{\mathrm{a}, *}$, Inneke Van Nieuwenhuyse ${ }^{\mathrm{a}}$

${ }^{a}$ Research Center for Operations Management, Department of Decision Sciences and Information Management, KU Leuven, Naamsestraat 69, 3000 Leuven, Belgium

\begin{abstract}
In many service systems, the arrival pattern is not constant throughout the day. This raises the question how staffing decisions should be adapted in view of controlling customer's waiting times. Assuming a single-stage queueing system with general abandonment and service times and time-varying demand for service, we suggest a method inspired by the simulation-based Iterative Staffing Algorithm (ISA) proposed by Feldman et al. (2008). The main advantage of our extension is that it enables to control the probability of experiencing an excessive waiting time, in particular in small systems.

Keywords: staffing, personnel planning, time-varying arrival rate, simulation, Iterative Staffing Algorithm
\end{abstract}

\section{Introduction}

In many service systems, the demand for service is not constant over time. Fluctuations on a daily, monthly, weekly or yearly basis can be present, which complicates the process of determining appropriate staffing

\footnotetext{
${ }^{*}$ Corresponding author.

Email addresses: mieke.defraeye@econ.kuleuven.be (Mieke Defraeye), inneke.vannieuwenhuyse@econ.kuleuven. be (Inneke Van Nieuwenhuyse)
} 
levels. Our particular focus lies on emergency departments (ED's): in these systems, capacity is the main lever to control waiting times, as customer service cannot be guaranteed by means of waiting lists or appointment systems. Determining capacity adequately in view of preserving customer service is a complex task, and as a result the capacity strategy in ED departments often mismatches demand ([28]). In an ED, service is mainly related to the length of the customer's waiting time (in particular the longest waits), and hence, controlling excessive waiting times is usually a primary goal. However, most approaches proposed in the literature focus on delay probabilities or expected waiting times.

This paper presents a simulation-based staffing method that enables to stabilize the probability of excessive waiting (i.e., the probability that the waiting time exceeds a maximum acceptable value) throughout the day. The suggested method is inspired by the Iterative Staffing Algorithm (ISA), proposed by Feldman et al. [7], which focuses on stabilizing the delay probability throughout the day (note that this corresponds to a maximum acceptable wait of zero). The use of discrete-event simulation provides distinct advantages over analytical methods, such as increased flexibility in modeling assumptions and the ability to control the precision of the results. The downside is that evaluation through simulation tends to be more time-consuming. Moreover, choosing appropriate staffing levels is further complicated due to the focus on excess wait probabilities: obtaining the probability of excessive waiting from the simulation's outcome is in general more difficult than calculating the delay probability. This research proposes a computationally efficient way to evaluate the probability of excessive waiting, and adjusts the staffing update function of the original ISA algorithm to account for the 
relatively small system size that characterizes an ED. Indeed, as opposed to call centers (which are commonly studied in the literature dealing with time-varying arrival rates), ED arrival rates tend to be rather low: [14] report hourly arrival rates ranging between 1 and 5 (in a New York ED); [27] describe a large urban hospital with hourly arrival rates varying between approximately 2 and 9 customers per hour.

While in reality, the ED resembles a multiserver queueing network in which patients move through several process steps to receive treatment, our approach considers the ED as a single-stage multiserver system with customer abandonments. Our experiments indicate that the proposed method (which we call $\operatorname{ISA}(\tau)$ ) succeeds in finding a staffing vector that meets the performance constraint, irrespective of system size. Large systems (for which the number of servers required is in the order of 100) and extremely small instances (requiring only 1-2 servers) can be solved, although the computation time increases with the problem size. A solution can be obtained for exponential as well as general service and abandonment time distributions, and staffing intervals are taken into consideration.

The remainder of the paper is organized as follows: in Section 2 we zoom in on the research problem. Relevant literature is discussed in Section 3: Section 3.1 discusses the stationary approximations that are most common our context, while Section 3.2 describes the Iterative Staffing Algorithm proposed in [7] which can be considered as the starting point of the $\operatorname{ISA}(\tau)$ approach we suggest. A detailed description of $\operatorname{ISA}(\tau)$ follows in Section 4 . Computational results of $\operatorname{ISA}(\tau)$ are reported in Section 5, and are compared to the staffing results obtained by applying stationary approximation techniques available in the literature. Section 6 summarizes our results, along 
with some directions for future research.

\section{Problem description}

We consider the ED as a single-stage multiserver $M(t) / G / s(t)+G$ queue, with time-varying arrivals and customer impatience (a schematic representation is given in Figure 1). Customers enter the system according to a Poisson pattern with time-varying arrival rate $\lambda(t)$ over the time horizon $[0, T]$ (the commonly used assumption of Poisson arrival patterns is supported by [1]). Only fluctuations on a daily basis are considered, as these tend to be most outspoken $([18])$. Customers may leave the system prematurely: the abandonment rate is denoted by $\theta$. Let $\mu$ denote the service rate of the service process, i.e. the rate at which one server (or physician) can treat customers (or patients). Once the treatment is finished, the patient leaves the system. Note that in reality, patients that need to be admitted to the hospital often have to wait after treatment until a bed becomes available (see e.g. [2]); as this waiting time results from factors other than the staffing decisions in the ED, we do not take it into account in our model. Contrary to the arrival process, the service and abandonment rates are assumed to be time-invariant and can follow any general distribution. The main goal is to determine an appropriate staffing requirement function $s(\tilde{t})$, which defines the number of servers (or physicians) to be assigned in each staffing interval $\tilde{t}$. The staffing function should guarantee that the excess wait probability (defined as the probability that an arriving customer's waiting time exceeds some maximum acceptable waiting time) is sufficiently low at all time instants. This can be expressed as:

$$
\operatorname{Pr}(W(t)>\tau) \leq \alpha \quad 0 \leq t<T
$$




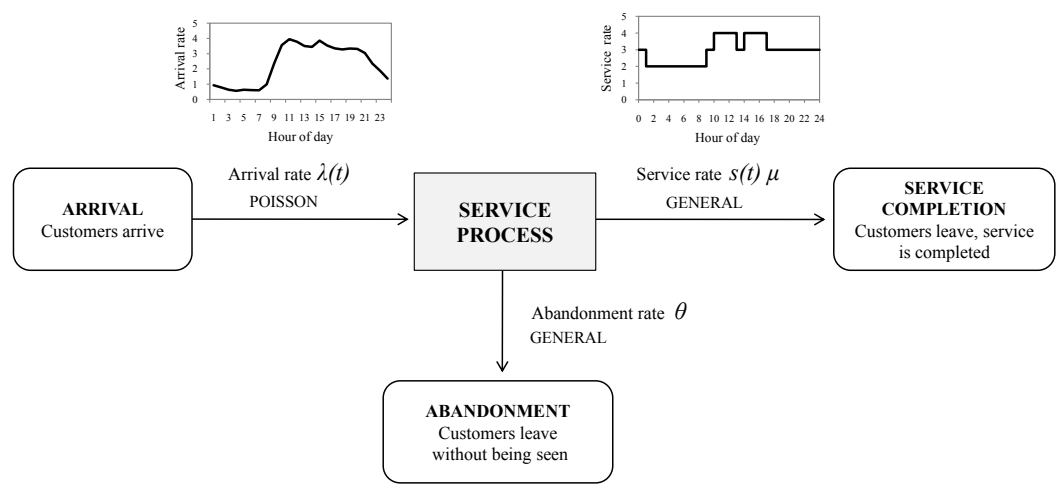

Figure 1: Schematic representation a single-stage queueing system with time-varying demand

where $W(t)$ represents the virtual waiting time ${ }^{1}$ of a customer arriving at time $t, \tau$ indicates the maximum acceptable waiting time, and $\alpha$ denotes the targeted (i.e. the maximum allowed) excess wait probability. We assume that $\tau$ is significantly smaller than the expected time-to-abandon (otherwise $\tau$ would probably be misspecified, especially in the context of an ED). Note that, by controlling excess wait probabilities, the number of abandoning customers is influenced as well. The number of servers is only allowed to change at fixed points in time; the planning period over which the number of servers is assumed to remain unchanged is referred to as the staffing interval (hence the distinction between time $t$ and staffing interval $\tilde{t}$ ).

Note that Expression 1 reduces to a constraint on the delay probability for $\tau=0$. Consequently, our performance requirement is more general than the (commonly used) delay probability. Measuring performance based on excess wait probabilities gives more leeway to the staffing level function,

\footnotetext{
${ }^{1}$ The virtual waiting time $W(t)$ is defined as the time that a customer would have to spend in queue if he were to arrive at time $t$ (cf. [16], pp. 13-14).
} 
while at the same time preserving patient service, as the decision maker can decide on the value of $\tau$.

\section{Related literature}

In this section, we briefly describe the literature that is most relevant to our research: we provide a description of the so-called stationary approximations (Section 3.1) and discuss the Iterative Staffing Algorithm (ISA) introduced in [7] (Section 3.2). For a more comprehensive overview of the literature on staffing systems with time-varying demand, the interested reader is referred to [15], [32], [4], and references therein.

\subsection{Stationary approximations}

The most common way to deal with the time-varying nature of the demand for service, is to approximate the non-stationary, time-varying system by one (or more) related stationary model(s). In Section 5 , the performance of $\operatorname{ISA}(\tau)$ is tested against a number of these approaches, so we prefer to briefly discuss them here.

In the Pointwise Stationary Approximation (PSA), the arrival rate at each time instant is plugged into a separate stationary model to obtain steady state performance measures for each moment in time ([10], [22], [29]). PSA is most appropriate in large systems with limited nonstationarity, characterized by high service rates, high targeted quality of service, and low to moderate loads.

To improve performance in case of low service rates, a lagged variant of PSA (denoted LagPSA) has been proposed ([11], [12]). This approach is identical to PSA, except for the use of a lagged arrival rate $\lambda(t-E[S])$, 
where $E[S]$ (i.e. the expected service time) represents a time lag (further theoretical background supporting this choice, can be found in [6]).

In the Modified Offered Load (MOL, [21],[25], [26]) approach, a stationary model is solved at each point in time, using a modified arrival rate that equals the product of the service rate and the infinite server offered load (i.e. the number of servers that would be used if infinitely many servers were available). As the number of servers declines, the MOL approximation becomes less accurate because of a lower resemblance to the infinite server system $([26])$.

The PSA, lagPSA and MOL approaches vary staffing levels continuously and do not account for the presence of staffing intervals. To this purpose, two refinements to the PSA approach have been proposed: Segmented PSA and Stationary Independent Period-by-Period (SIPP). In the Segmented PSA approach (see e.g. [15]), the staffing levels are set equal to the maximum of the PSA staffing requirements over the staffing interval ([15]). The SIPP approach $([12],[14])$ uses a stationary model in each staffing interval, with the arrival rate averaged over that interval. As shown in [12], SIPP does not perform well when staffing intervals are long or when the arrival rate changes substantially over the staffing interval.

Further refinements to the SIPP approach have been proposed in [12] and [13]: Lag SIPP (which uses a lagged arrival rate), SIPPmax (which uses the maximum arrival rate over the staffing interval instead of the average, and hence coincides with Segmented PSA), and Lag SIPPmax (which is a combination of both). As shown in [13], Lag SIPP and Lag SIPPmax tend to have better performance than SIPP and SIPPmax. 
Once the nonstationary system has been transformed into one or more stationary models, approximations are often needed to obtain the steady state performance measures, in particular when service times and abandonment times are generally distributed (as in our setting). For further details on approximations for the $M / G / s+G$ model, we refer to [20] and [31].

Often however, explicit performance calculations are avoided by using a rule of thumb, known as the square-root staffing rule (SRS; see [8], [30] and [23]). The main benefit of SRS lies in its simplicity and robustness: at each time instant, staffing is determined as the offered load (denoted $m$ ) augmented with an amount of safety capacity (cf. Expression 2). The required safety capacity is proportional to the offered load, and depends on the desired quality of service (which is reflected in the quality of service parameter $\beta$ ):

$$
s=m+\beta \sqrt{m}
$$

The appropriate $\beta$ can be obtained through the inverse of the Halfin-Whitt delay function (for $M / M / s$ models, cf. [17]) or the Garnett delay function (for $M / M / s+M$ models, cf. [9]). An extension towards $M / M / s+G$ models can be found in [33]. As a rule of thumb, the SRS rule is easy to apply. However, it provides no firm guarantee that the desired performance constraint is actually met.

As stated in [12], the use of stationary models implicitly assumes that: (1) delays between separate intervals are statistically independent; (2) steady state is reached in each interval, (3) the arrival rate remains constant over the staffing interval, and (4) no overloading is present within any given interval, as this would cause instability in the stationary model. These assumptions 
are unlikely to hold in an ED context, as an ED represents a system that is often temporarily overloaded (violating assumption 4) and where substantial changes in the arrival rate during the staffing interval are common (violating assumption 3). Moreover, due to the combination of typically large service times and rather short staffing intervals, assumptions 1 and 2 are likely to be violated as well.

\subsection{The Iterative Staffing Algorithm (ISA)}

In [7], a promising simulation-based technique for determining staffing requirements in time-varying queues is proposed. As the name suggests, the Iterative Staffing Algorithm (ISA) repeatedly evaluates and alters the staffing function, until the desired performance is attained. For each staffing function, system performance is evaluated by means of simulation and the staffing level is updated based on the observed performance. This sequence of evaluating performance and updating staffing levels is called an iteration.

Performance is expressed in terms of a constraint on the delay probability, that is, the delay probability must lie below a target value $\alpha$ at all time instants:

$$
\operatorname{Pr}(W(t)>0) \leq \alpha \quad 0 \leq t \leq T
$$

Equivalently, the delay probability equals the probability that the number of customers in the system $N(t)$ surpasses the available capacity $s(\tilde{t})$, leading to the following constraint:

$$
\operatorname{Pr}(N(t) \geq s(\tilde{t} \mid t \in \tilde{t})) \leq \alpha \quad 0 \leq t \leq T
$$


The ISA assumes staffing changes can be made almost continuously. The planning horizon $T$ is divided into very small intervals: staffing changes are only allowed at the start of each interval and the number in system is evaluated once every staffing interval. ISA then proceeds as follows. Initially, all staffing levels are set equal to an arbitrarily large number. Subsequently, system performance is simulated by performing a fixed number of independent replications, which results in a distribution of the number of customers in system at each moment in time. Then, staffing levels are improved (simultaneously for all staffing intervals) such that in each staffing interval $\tilde{t}$, the staffing level corresponds to the smallest value of $s(\tilde{t})$ satisfying the performance requirement in Expression 4. Formally, the evaluation of the distribution of the number of patients in system at the start of staffing interval $\tilde{t}$ in iteration $i$ (denoted $N_{i}(\tilde{t})$ ) determines the staffing level in interval $\tilde{t}$ in iteration $i+1\left(\right.$ denoted $\left.s_{i+1}(\tilde{t})\right)$ :

$$
s_{i+1}(\tilde{t})=\arg \min \left\{k \in \mathbb{N}: \operatorname{Pr}\left(N_{i}(\tilde{t}) \geq k\right) \leq \alpha\right\} \quad \forall \tilde{t}
$$

The algorithm stops when the staffing changes in subsequent iterations become sufficiently small for all staffing intervals (i.e., staffing levels differ by at most 1 , for all $\tilde{t}$ ).

The major advantage of the ISA lies in the use of simulation to evaluate system performance. As a result, the appropriateness of the staffing function generated by ISA is validated automatically (that is, under the assumption that the simulation model is adequate). Moreover, the method has potential to be applied to general settings, for which analytical results are no longer available. However, some aspects make the traditional ISA less appropriate 
in an $\mathrm{ED}$ context:

- Firstly, an ED is commonly characterized by a rather small system size; arrival rates tend to be much lower than in the examples provided in [7]. As discussed in the original publication, the delay probabilities obtained by the original ISA tend to be less stable in periods with low demand, as even a small change in capacity has a substantial impact on performance. Moreover, the conventional stopping rule of the ISA might pose problems: the algorithm stops when the change in staffing requirements is at most 1 unit in all staffing intervals and thus staffing changes of $+/-1$ server are disregarded. In small systems however, the addition or removal of one server can result in substantial differences in performance.

- Secondly, the ISA does not explicitly deal with the length of the staffing intervals, i.e. the time period over which capacity remains constant (all examples used in [7] assume small staffing intervals with a length of $0.1 / \mu$ ). As the number of customers in system is measured only once every interval, it can be expected that an increase in the staffing interval length will lead to a decrease in accuracy, which will negatively impact the algorithm's performance. In the method we present in Section 4, this problem is addressed by making a distinction between staffing intervals and (smaller) calculation intervals.

- Finally, the results in [7] indicated that a staffing function that stabilizes delay probability does not automatically stabilize other performance measures (such as abandonment probabilities, average queue lengths and average waiting times). As discussed in Section 2, the delay probability is not the most appropriate performance measure in an ED environment, as most patients are able to tolerate a (small) 
waiting time. Focusing on the probability of excessive waiting is more relevant and allows more flexibility, as the decision maker can decide both on the maximum acceptable waiting time that should be met, and on the service level $\alpha$ (see Expression 1). Focusing on excess wait probabilities, however, implies that staffing levels can no longer be set using Expression 5.

These observations justify the search for a method which (1) is suitable for very small system sizes such as an ED, (2) is capable of dealing with staffing intervals over which the capacity remains constant and (3) emphasizes low probabilities of excessive wait rather than delay probabilities. In the next sections, a more detailed description of the $\operatorname{ISA}(\tau)$ method is given.

\section{4. $\operatorname{ISA}(\tau)$ algorithm}

\subsection{Notation}

Let $s_{i}(\tilde{t})$ represent the staffing level in staffing interval $\tilde{t}$ at the start of $i^{\text {th }}$ iteration of the algorithm, so that $\mathbf{s}_{i}$ denotes the staffing vector. The performance vector corresponding to $\mathbf{s}_{i}$ is represented as $\mathbf{P}_{i}$; its elements $P_{i}(t)$ denote the excess wait probability for a customer arriving at the start of each calculation interval $t$. The squared coefficient of variation of $\mathbf{P}_{i}$ will be represented by $S C V_{i}$. For a given staffing interval $\tilde{t}$ starting at $t_{s}$ and ending at $t_{f}, P_{\max , i}(\tilde{t})$ denotes the maximum value of the excess wait probabilities $P_{i}(t)$ that directly result from the capacity $s_{i}(\tilde{t})$ (as detailed below in Section 4.2 , this implies $t \in\left[t_{s}-\tau, t_{f}-\tau\left[\right.\right.$ ). Each vector $\mathbf{P}_{i}$ (containing excess wait probabilities per calculation interval $t$ ) can thus be translated into vector $\mathbf{P}_{\text {max }, i}$ (containing maximum excess wait probabilities per staffing interval $\tilde{t})$. For a given staffing vector $\mathbf{s}_{i}, e_{i}$ denotes the number of staffing intervals 
for which the performance target is not met (i.e. $P_{\max , i}(\tilde{t})>\alpha$ ). Additionally, we associate a cost $c_{i}$ (expressed in man-hours) with each staffing vector $\mathbf{s}_{i}$, and define $c^{*}$ to be the lowest cost found so far, for a solution that meets the performance constraint at all times. The cost (expressed in man-hours) associated to using one unit of capacity during one staffing interval is represented as $u$.

\subsection{Procedure}

The $\operatorname{ISA}(\tau)$ algorithm proceeds as follows: initially, the capacity in each staffing interval is set equal to the offered load that would prevail if the system were stationary, namely the ratio of the average arrival rate over the time horizon to the service rate (rounded up, since capacity is integer). A first phase in the algorithm (referred to as PHASE I, and summarized in Algorithm 1) aims at quickly finding a staffing vector for which performance is close to the target (but not necessarily below the target value at all times). To this purpose, the current staffing level function $s_{i}(\tilde{t})$ is altered iteratively, based on the simulation output.

During each iteration $i$ of the algorithm, $s_{i}(\tilde{t})$ is updated as follows:

$$
s_{i+1}(\tilde{t})=\left\{\begin{array}{lll}
\left\lceil s_{i}(\tilde{t}) A_{i}(\tilde{t})\right\rceil & \text { if } A_{i}(\tilde{t}) \geq 1, & \forall \tilde{t} \\
\left\lfloor s_{i}(\tilde{t}) A_{i}(\tilde{t})\right\rfloor & \text { if } A_{i}(\tilde{t})<1, & \forall \tilde{t}
\end{array}\right.
$$

where $A_{i}(\tilde{t})$ refers to an amplification factor, which is determined based on the deviation between $P_{\max , i}(\tilde{t})$ and the target $\alpha$ (in percent):

$$
A_{i}(\tilde{t})=1+\frac{P_{\max , i}(\tilde{t})-\alpha}{\alpha i} \quad \forall \tilde{t}
$$

$P_{\max , i}(\tilde{t})$ is derived from the simulation results, using the evaluation method 
described in [3]. For each staffing interval $\tilde{t}$ (starting at $t_{s}$ and ending at $t_{f}$ ), $P_{\max , i}(\tilde{t})=\max \left\{P_{i}(t): t \in\left[t_{s}-\tau, t_{f}-\tau[\}\right.\right.$. Indeed, if a capacity shock occurs at $t_{s}$, the excess wait probabilities are affected for all arrivals after $\left(t_{s}-\tau\right)$, as $\tau$ represents the time window within which these patients should start service in order to have a waiting time below the maximum wait (as illustrated in Figure 2). Thus, the capacity in a staffing interval $\tilde{t}$ (starting at $t_{s}$ and ending at $t_{f}$ ) has a direct ${ }^{2}$ effect on the performance of patients arriving during interval $\left[t_{s}-\tau, t_{f}-\tau[\right.$.

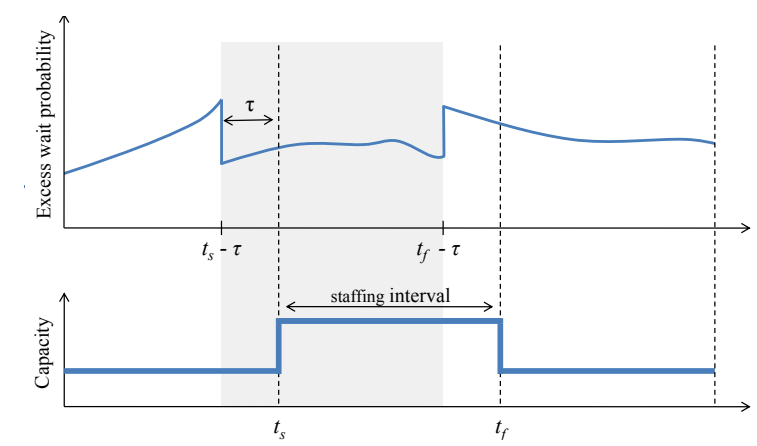

Figure 2: Interval over which capacity impacts performance

Excess wait probabilities below (above) target will result in an $A_{i}(\tilde{t})$ below (above) 1 and thus a decrease (increase) in capacity in the corresponding interval (note that due to the rounding in Expression 6, capacity is always increased or decreased with at least one unit).

The use of the scaling factor $i$ in the denominator of Expression 7 ensures that $A_{i}(\tilde{t})$ approaches $1(\forall \tilde{t})$ as the number of iterations increases. This forces the algorithm to decrease the size of the staffing changes as it

\footnotetext{
${ }^{2}$ It is clear that an indirect effect is present as well; i.e. the capacity level in any staffing interval also has an impact on the performance at all later time instants, through the number of customers in system.
} 


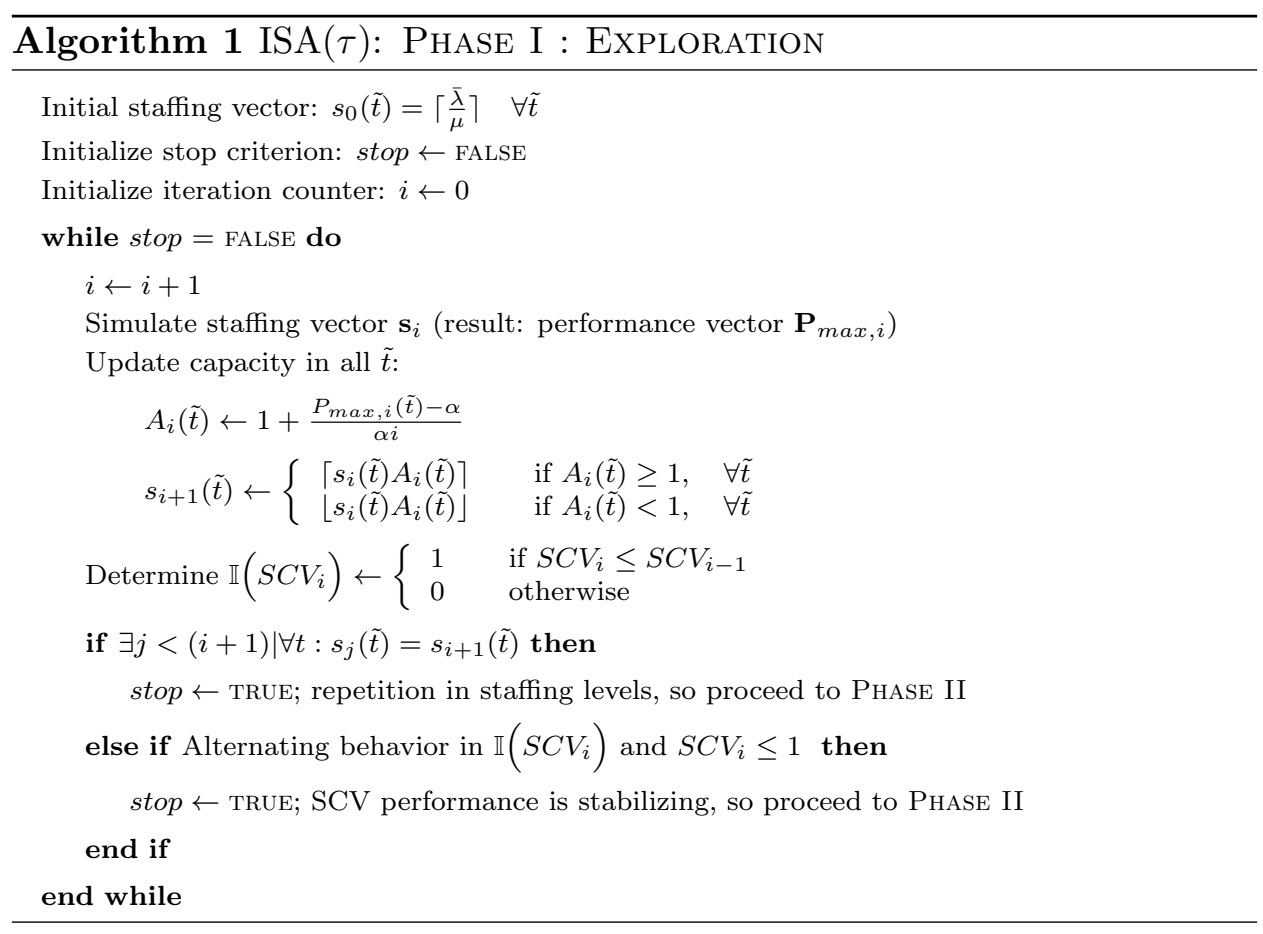

progresses, eventually switching to unit-size changes (and, in the limit, converging to a final staffing vector despite the fact that possible deviations from the target may still remain). In fact, the choice of the scaling factor in the denominator of Expression 7 is rather arbitrary; other factors may be considered, such as $i / 2$ or $i^{2}$. Especially in large systems, high values for the factor should be used with caution: they may lead the algorithm to switch to unit-size capacity changes too soon, causing the number of iterations in the exploration phase to increase substantially. This issue is illustrated numerically in Section 5.1.

We allow the algorithm to stop exploring when either (1) cycling occurs (meaning that the staffing vector put forward in the current iteration has already been assessed during a previous iteration), or (2) when the excess 
wait probability is stabilizing ${ }^{3}$. To check condition (2), we keep track of the squared coefficient of variation of the excess wait probability over the time horizon in each iteration $i$ (denoted as $S C V_{i}$ ), and record whether it increases or decreases with respect to the previous iteration (denoted as $\mathbb{I}\left(S C V_{i}\right)=1$ if $S C V_{i} \leq S C V_{i-1}, 0$ otherwise). The algorithm stops when $S C V_{i}$ is sufficiently low (we obtained good results with the criterion $S C V_{i} \leq 1$ in our experiments) and a cycle occurs in $\mathbb{I}\left(S C V_{i}\right)$, signaling that $S C V_{i}$ starts alternating between increase(s) and decrease(s) for subsequent iterations without any significant improvement. Though condition (2) is more ad hoc, it is particularly useful in large systems: we observed that although many iterations are needed before cycling in the staffing levels occurs (i.e. before condition (1) is met), $S C V_{i}$ usually stabilizes far more quickly. An illustration of the typical evolution of $S C V_{i}$ throughout the algorithm in a large problem setting is given in Figure 3. As such, criterion (2) can substantially lower the computational time in PHASE I for large systems.

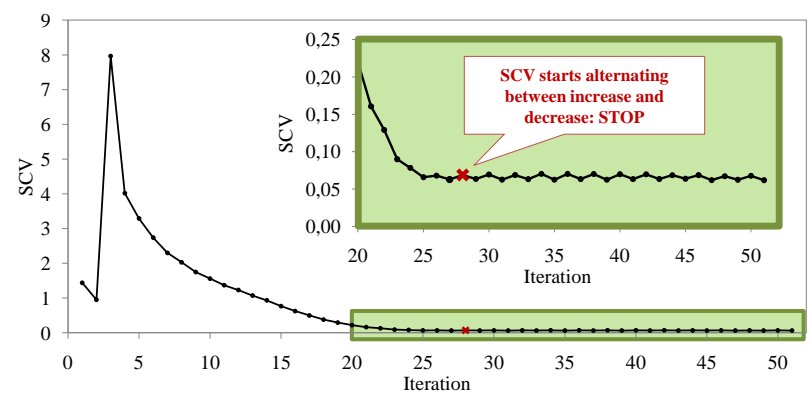

Figure 3: Evolution $S C V_{i}$

\footnotetext{
${ }^{3}$ Recall that the original ISA uses a different terminating condition: it stops when the staffing level changes at most with one unit in each interval, compared to the previous iteration. We opt not to use this stop criterion due to the focus on small system sizes, where one unit capacity changes may occur more frequently (causing the algorithm to stop prematurely).
} 
Note that the exploration phase does not necessarily result in a feasible staffing vector. Consequently, an additional fine-tuning procedure (PHASE II or "exploitation phase", detailed in Algorithm 2) is needed. The aim of PHASE II is to deduce feasible solutions from the infeasible staffing levels obtained in PHASE I, in hope of finding a solution which outperforms the best feasible solution found so far (if any) in terms of labor cost. To that end, all infeasible solutions encountered during PHASE I are first sorted based on increasing maximum excess wait probability over the time horizon (i.e. increasing values of $\max _{\tilde{t}}\left\{P_{\max , k}(\tilde{t})\right\}$, where index $k$ denotes an infeasible solution). Indeed, a lower value indicates smaller deviations from the target, which makes the corresponding solution more promising to fine-tune. In case of a tie, the projected staffing cost (expressed in man-hours) is considered, i.e. the staffing cost that results when adding 1 unit of capacity to each staffing interval that causes the excess wait probability to surpass the target. Indeed, if this target is exceeded in just a limited number of intervals, a small number of capacity increases may be sufficient to obtain a feasible staffing level vector, which is appealing from a labor cost perspective.

Next, all infeasible solutions are improved one by one (in sorted order). For a given infeasible solution $j$, an improved staffing vector $\mathbf{s}_{j}^{\prime}$ (with corresponding $\operatorname{cost} c_{j}^{\prime}$ ) is constructed by adding one unit of capacity in all staffing intervals where performance is unsatisfactory. The new staffing vector's performance is evaluated through simulation, provided that its cost is strictly lower than $c^{*}$ (further exploiting the current infeasible solution is futile if $\left.c_{j}^{\prime} \geq c^{*}\right)$. Based on the simulation results, two cases can be distinguished:

- The performance constraint is not yet met, in which case the exploitation continues. Vector $\mathbf{s}_{j}^{\prime}$ is improved further (unit size capacity in- 
creases are made) and its performance is simulated (if the cost is lower than $\left.c^{*}\right)$.

- The performance constraint is satisfied at all times; in this case a new feasible solution is found, which is stored if it is less costly than the current best feasible solution in terms of labor cost. The exploitation of solution $j$ is terminated and the algorithm then proceeds to the next infeasible solution in the sorted list.

Note that the procedures described in PHASE I and PHASE II are suitable for small system sizes, largely avoid cyclic behavior and moreover guarantee that the algorithm yields a staffing vector meeting the performance constraint.

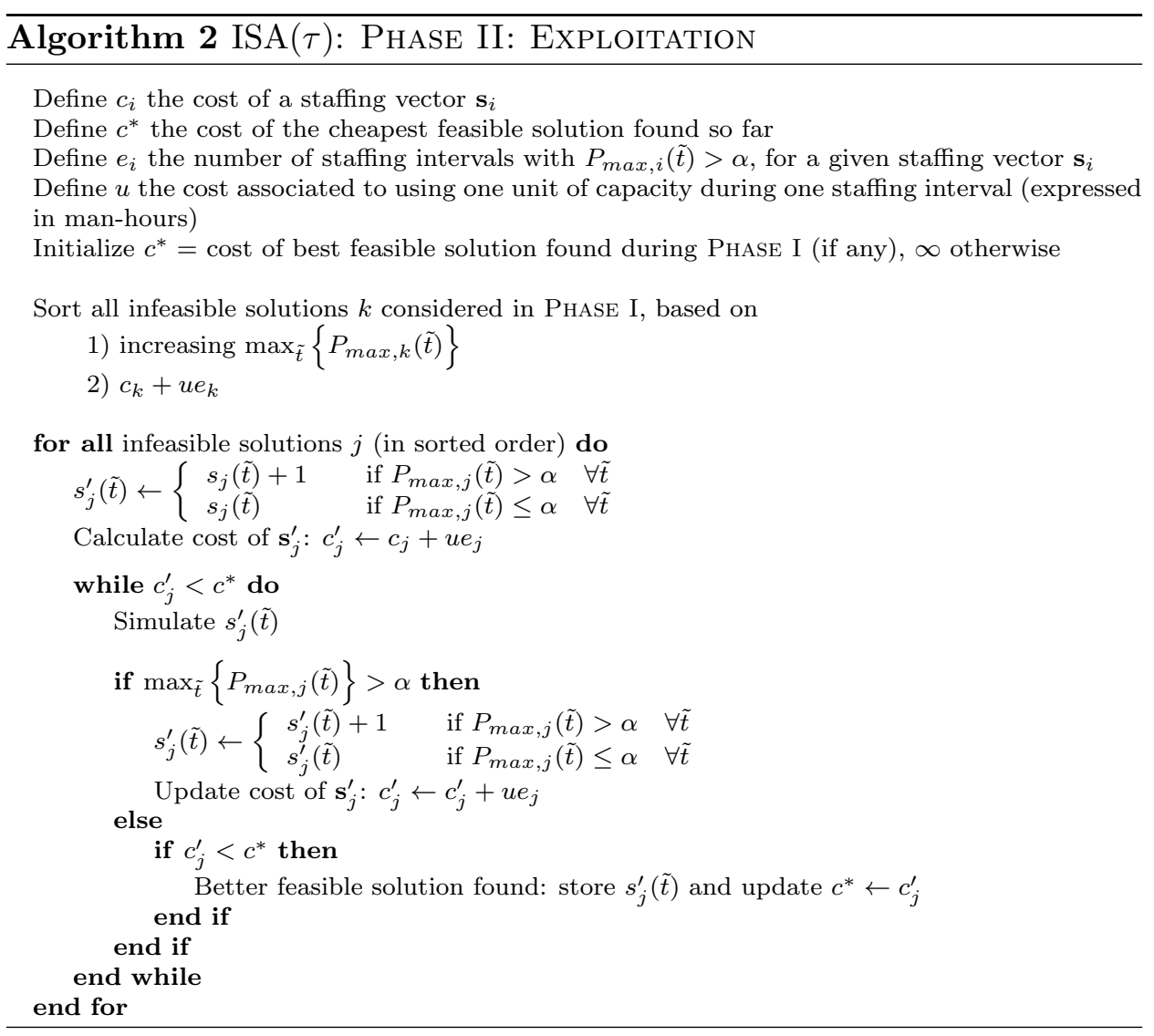




\section{Computational results}

The method we proposed in Section 4 was tested for two settings: a largescale example taken from [7] (we added the assumption of 15 minute staffing intervals), and a small-scale setting based on real-life arrival data at the ED of a Belgian hospital. Unfortunately, no detailed process data were available from the hospital, hence we assume service times that are exponentially distributed with mean 30 minutes (similar to Green et al. (2006), where service times were chosen based on physician workload estimations that are available in Graff et al. (1993)). Table 1 summarizes the main characteristics for both examples; in Figure 4 the corresponding arrival rates are plotted (note that the example derived from [7] represents a large scale system, with arrival rates varying between 80 and 120 customers per hour). For both examples, a 24-hour time horizon is considered and performance was calculated quasi-continuously: the calculation interval length is set equal to 1 minute. The length of the staffing interval equals 15 minutes and the maximum acceptable waiting time $\tau$ is set to 10 minutes. Per iteration of the algorithm, 2500 replications are performed. The algorithm's performance for the 2 examples is first evaluated in a $M(t) / M / s(t)+M$ context in Section 5.1. However, the algorithm remains applicable in a $M(t) / G / s(t)+G$ context, as the experiments in Section 5.2 indicate. In Section 5.3, we compare the proposed $\operatorname{ISA}(\tau)$ method with some heuristics available in the literature.

\subsection{ISA $(\tau)$ : Exponential service and abandonment times}

A comparison of the results for both small and large settings in an $M(t) / M / s(t)+M$ setting (see Table 2), leads to the conclusion that our algorithm results in staffing levels that indeed meet the desired performance targets in relatively few iterations. That is, a staffing vector is found for 


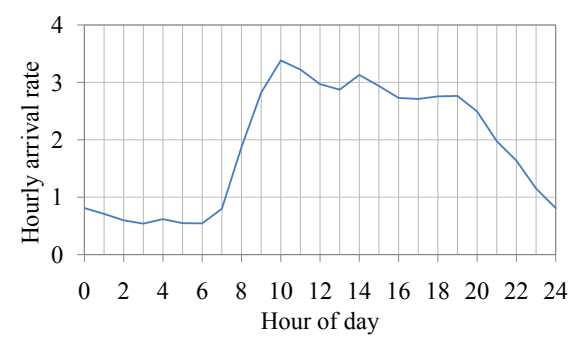

(a)

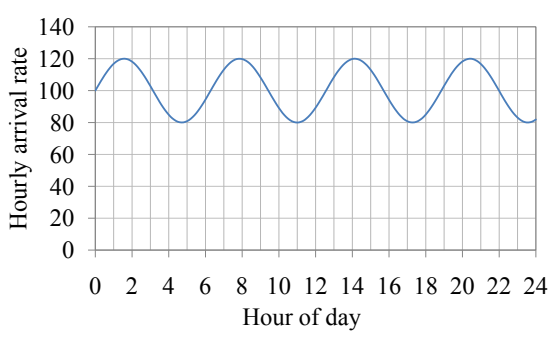

(b)

Figure 4: Arrival rate (a) Belgian hospital (b) example based on [7]

\begin{tabular}{lcc}
\hline & Belgian hospital & Example based on [7] \\
\hline Service rate (customers/hour) & 2 & 1 \\
Abandonment rate (customers/hour) & 0.25 & 1 \\
\hline Time horizon & 24 hours \\
Calculation interval & 1 minute \\
Staffing interval & 15 minutes \\
Maximum acceptable wait $\tau$ & 10 minutes \\
Target excess wait probability $\alpha$ & 0.1 \\
Performance constraint & $\operatorname{Pr}(W(t)>\tau) \leq \alpha \quad \forall t$ \\
Number of replications (per iteration) & 2500 \\
\hline
\end{tabular}

Table 1: System parameters

which the probability of excessive wait lies below the chosen acceptable waiting time threshold and does not depend on the customer's arrival time. However, the number of iterations needed increases with system size: small systems (which we focus on) require less iterations than large systems. Moreover, as the capacity shocks are more frequent and larger in size in the large scale system (see Figure 6), so are the performance shocks (for both excess wait probability and delay probability). Finally, the performance graphs in Figures 5 and 6 clearly indicate that the delay probability is indeed a more restrictive performance measure; therefore, overstaffing might occur if the delay probability is used as a performance constraint (assuming that 
customers tolerate a waiting time of $\tau>0$ ).

\begin{tabular}{lcc}
\hline & Belgian hospital & Example based on [7] \\
\hline Number iterations Phase I & 6 (no feasible) & 17 (no feasible) \\
Number iterations Phase II & 5 (3 feasible) & 6 (2 feasible) \\
$\max _{t}\{P(t)\}$ & 0.090 & 0.100 \\
Staffing cost & 74 & 2257.25 \\
\hline
\end{tabular}

Table 2: Results $\operatorname{ISA}(\tau)$ : exponential service and abandonment times
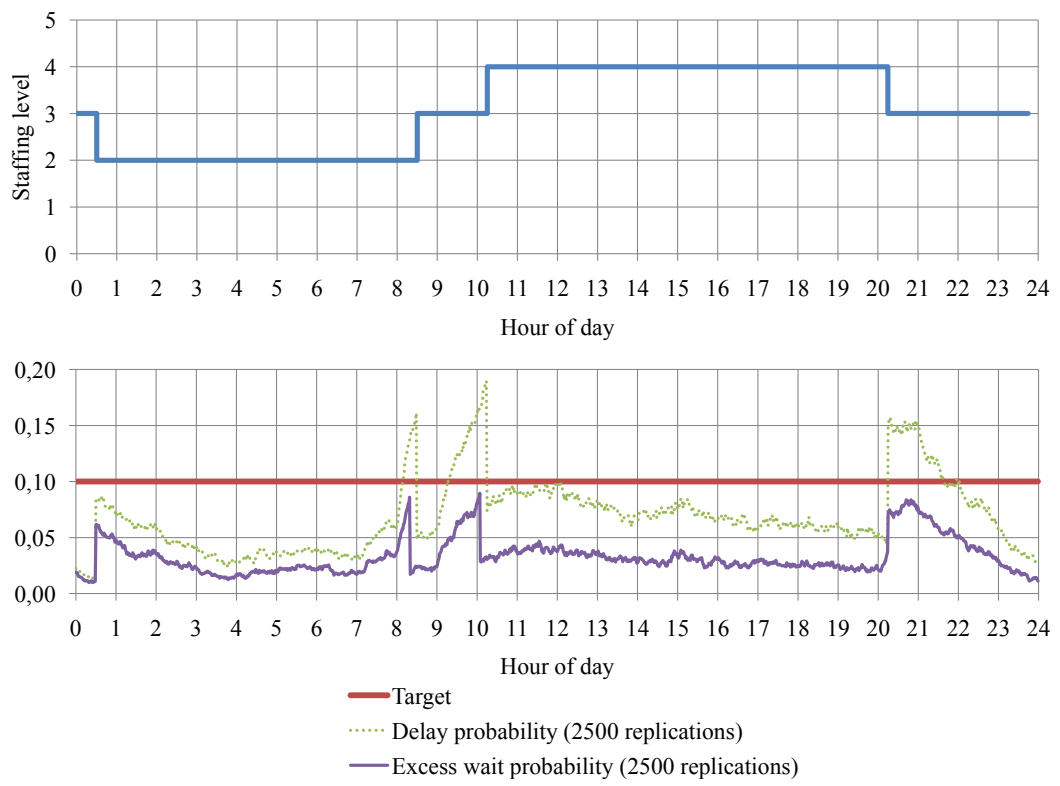

Figure 5: Small system: staffing vector and resulting performance (exponential service and abandonment times)

As mentioned in Section 4.2, the speed of convergence in the exploration phase can be influenced by changing the scaling factor $i$ in Expression 7 . Appendix A compares the results for both systems, using alternative scaling factors. As evident from these results, the number of iterations in the exploration phase increases drastically when the algorithm is forced to move 

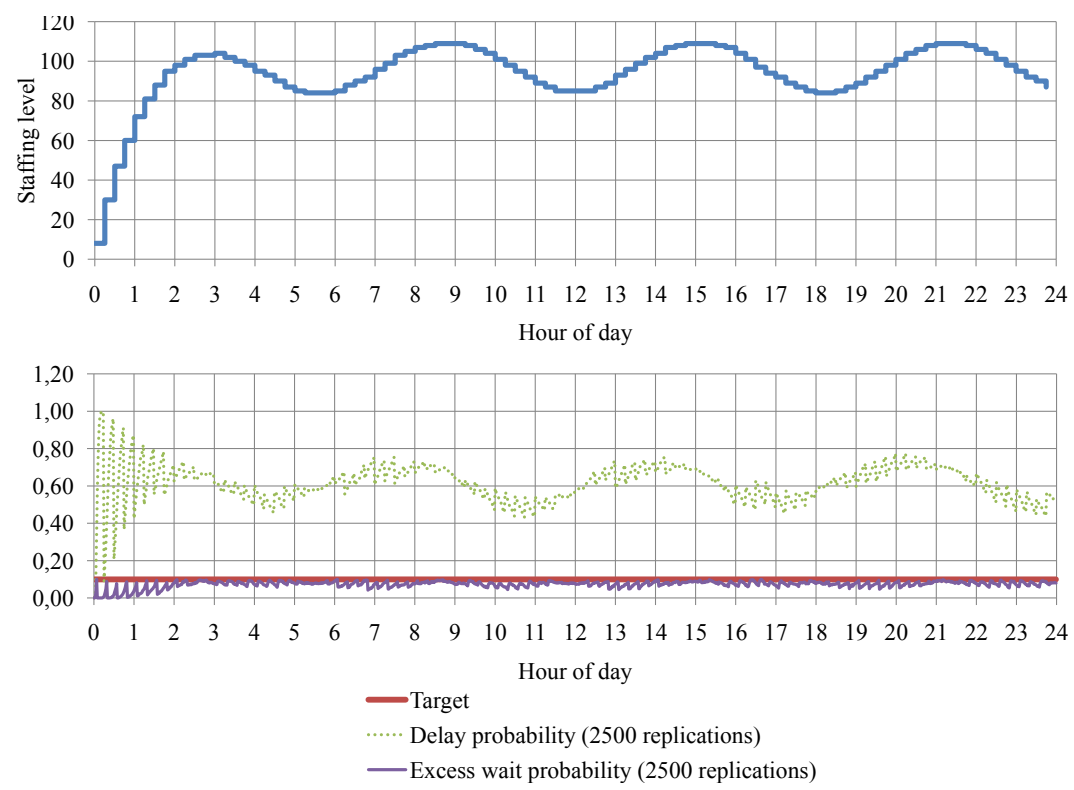

Figure 6: Large system: staffing vector and resulting performance (exponential service and abandonment times)

to unit-size capacity changes too soon (e.g. when a factor $i^{2}$ is used). Moreover, there is no evidence that this decrease in efficiency in the exploration phase pays off in the exploitation phase, nor does it seem to impact the quality of the final solution (both in terms of excess wait probability and staffing cost).

\subsection{Lognormal service and abandonment times}

One of the major advantages of a simulation-based method is its general applicability. As such, experiments were repeated assuming an $M(t) / G / s(t)+$ $G$ setting, assuming service and abandonment times are lognormally distributed with squared coefficient of variation (SCV) equal to 1.5 and 0.5. As the results in Table 3 indicate, the algorithm's effectiveness does not change when departing from the exponential service time and abandonment 
time distributions. Also, the number of iterations needed by the algorithm does not change substantially.

\begin{tabular}{lcccc}
\hline & \multicolumn{2}{c}{$\begin{array}{c}\text { Small system } \\
(\mu=2)\end{array}$} & \multicolumn{2}{c}{$\begin{array}{c}\text { Large system } \\
(\mu=1)\end{array}$} \\
& $\mathrm{SCV}=0.5$ & $\mathrm{SCV}=1.5$ & $\mathrm{SCV}=0.5$ & $\mathrm{SCV}=1.5$ \\
\hline Number iterations PHASE I & 6 & 5 & 13 & 22 \\
Number iterations PHASE II & 5 & 4 & 16 & 6 \\
$\max _{t}\{P(t)\}$ & 0.090 & 0.083 & 0.099 & 0.099 \\
Staffing cost & 73.25 & 74.5 & 2492.25 & 2363.4 \\
\hline
\end{tabular}

Table 3: Results ISA $(\tau)$ : Lognormal service and abandonment times

\subsection{Comparison to other staffing heuristics}

In this section, the staffing vector obtained by $\operatorname{ISA}(\tau)$ is compared to some readily implementable staffing heuristics based on the stationary approximations as discussed in Section 3.1. The key features of the selected heuristics are summarized in Table 4.

The SRS_SIPPmax, SRS_lagSIPPmax and SRS_MOL heuristics are all based on the square root staffing rule (SRS). The value for $\beta$ is determined using the Garnett delay function ([9]). The CF_MOL heuristic is based on the available exact results for stationary $\mathrm{M} / \mathrm{M} / \mathrm{s}$ models (i.e. without abandonments): at each point in time, the closed form formula for the excess wait probability (cf. [16] pp. 66-72) is used to assess the performance corresponding to a staffing vector. Again, a MOL approximation is used to capture the time-varying nature of the system. As the MOL approximation (used for SRS_MOL and CF_MOL) is calculated at each time $t$, the staffing level in a given staffing interval $\tilde{t}$ is set equal to the maximum staffing requirement over the interval. 


\begin{tabular}{|c|c|c|c|}
\hline Abbreviation & Staffing by means of & Applied to & $\begin{array}{l}\text { Used arrival rate } \\
\text { or offered load }\end{array}$ \\
\hline CF_MOL & Closed form formula $M / M / s$ & $\begin{array}{l}\text { each time } \\
\text { instant } t\end{array}$ & MOL arrival rate \\
\hline SRS_SIPPmax & $\begin{array}{c}\text { SRS formula } M / M / s+M \\
\text { (using Garnett delay function[9]) }\end{array}$ & $\begin{array}{l}\text { each staffing } \\
\text { interval } \tilde{t}\end{array}$ & $\begin{array}{l}\text { SIPPmax } \\
\text { offered load }\end{array}$ \\
\hline SRS_lagSIPPmax & $\begin{array}{c}\text { SRS formula } M / M / s+M \\
\text { (using Garnett delay function[9]) }\end{array}$ & $\begin{array}{l}\text { each staffing } \\
\text { interval } \tilde{t}\end{array}$ & $\begin{array}{l}\text { lagged SIPPmax } \\
\text { offered load }\end{array}$ \\
\hline SRS_MOL & $\begin{array}{c}\text { SRS formula } M / M / s+M \\
\text { (using Garnett delay function[9]) }\end{array}$ & $\begin{array}{l}\text { each time } \\
\text { instant } t\end{array}$ & MOL offered load \\
\hline
\end{tabular}

Table 4: Other heuristics available in the literature

Each of the four heuristics was applied to both the small and large system (cf. Table 1). Figures 7 and 8 show the corresponding staffing vectors and the resulting excess wait probabilities for the large system; Figures 9 and 10 show the results for the small system (assuming exponential service and abandonment times). In each case, the results are compared to those of $\operatorname{ISA}(\tau)$.

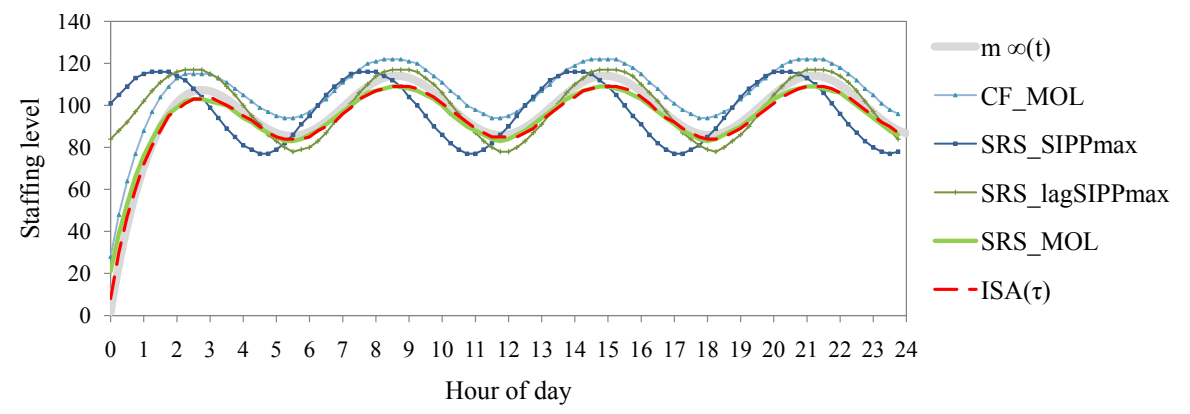

Figure 7: Comparison ISA $(\tau)$ staffing vs. other heuristics: Staffing (large system, exponential service and abandonment times)

The obtained staffing levels for the large system (represented in Figure 7) clearly illustrate the importance of the time lag between the arrival rate and the offered load. For SRS_SIPPmax, which does not account for this time lag, the peak staffing levels clearly occur earlier than the peaks in offered 

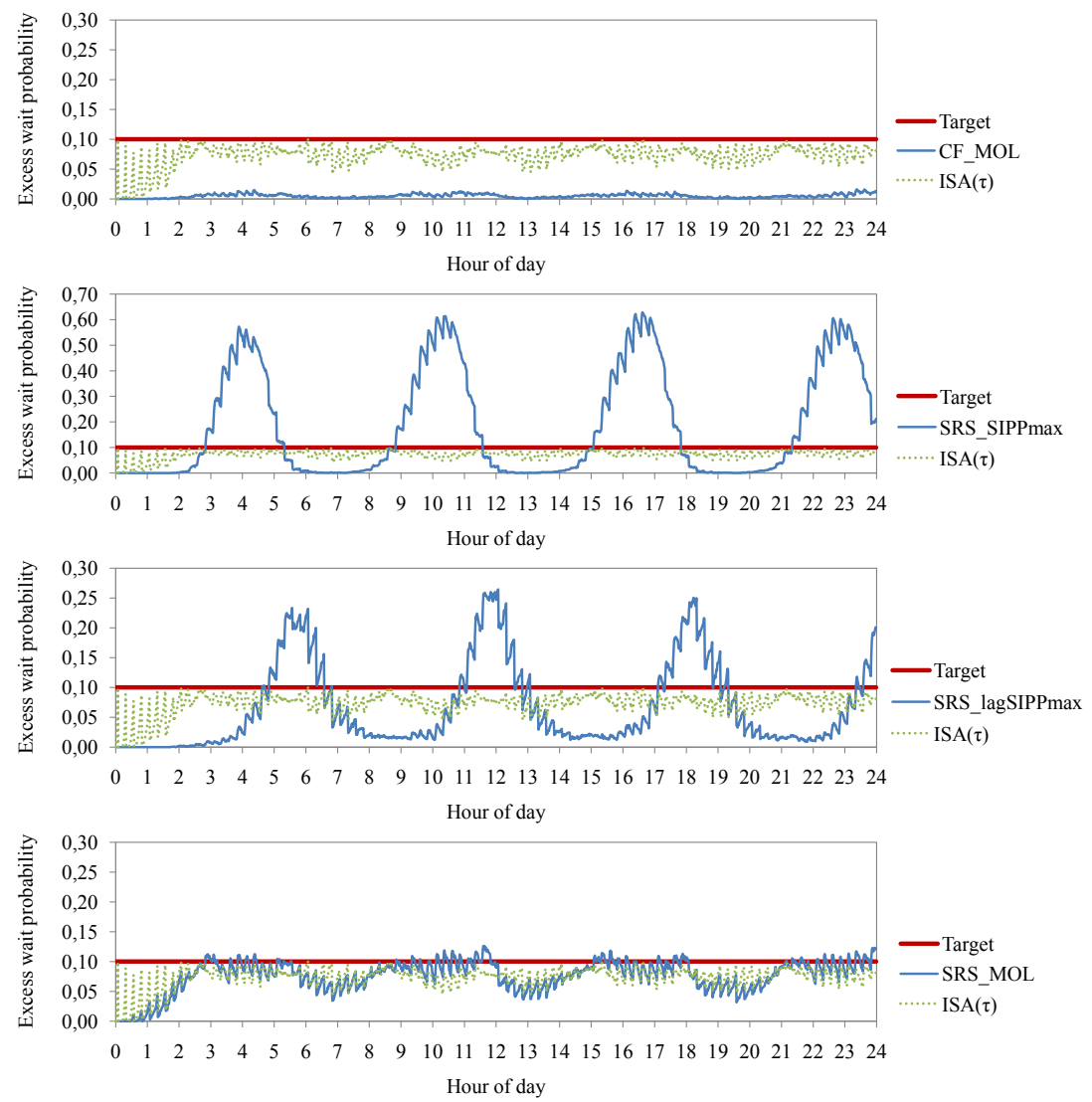

Figure 8: Comparison $\operatorname{ISA}(\tau)$ staffing vs. other heuristics: Performance (large system, exponential service and abandonment times)

load, leading to extremely poor performance. The time-varying nature of the offered load is captured better if a time lag is added (cf. SRS_lagSIPPmax) or if the MOL-approximation is used (cf. CF_MOL and SRS_MOL).

The observed performance for CF_MOL in the large system (represented in Figure 8) indicates that it leads to overstaffing, a result that can be explained by the fact that the underlying closed form $M / M / s$ formula ignores the presence of abandonments. For the large system, the SRS_MOL heuristic performs pretty well. This is not surprising, as the offered load used in the 
SRS_MOL heuristic is exact ${ }^{4}$ and in addition the conditions for the Garnett delay function are met (i.e., exponential service and abandonment times and a sufficiently large number of servers). For the large system, the SRS_MOL staffing vector yields the best results among all stationary approximations. The excess wait probability occasionally exceeds the target though, which might be explained in part by the presence of staffing intervals, and by the fact that the SRS rule is a rule of thumb and therefore provides no guarantee for the performance constraint being met. The excess wait probabilities obtained through ISA $(\tau)$ closely resemble those of SRS_MOL, yet for ISA $(\tau)$ the performance constraint is met at all times.

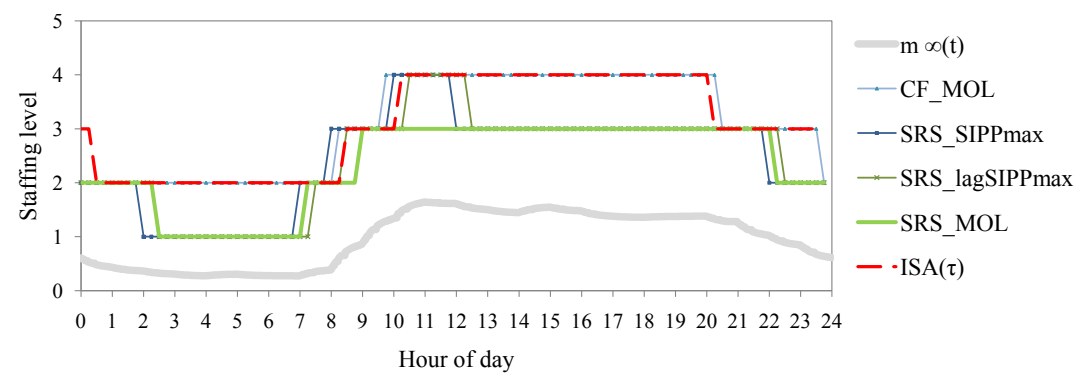

Figure 9: Comparison ISA $(\tau)$ staffing vs. other heuristics: Staffing (small system, exponential service and abandonment times)

The results for the small example setting (given in Figures 9 and 10) show that none of the SRS-based heuristics result in adequate staffing. This might be addressed to the SRS rule performing best for moderate to large offered loads [9], whereas we applied it to a very small system. The closed-

\footnotetext{
${ }^{4}$ The distribution of number in system in any $M(t) / G / s(t)+G$ system is identical to that of the infinite server model, if the specific condition holds that the abandonment rate is equal to the service rate [32], as is the case in this setting.
} 

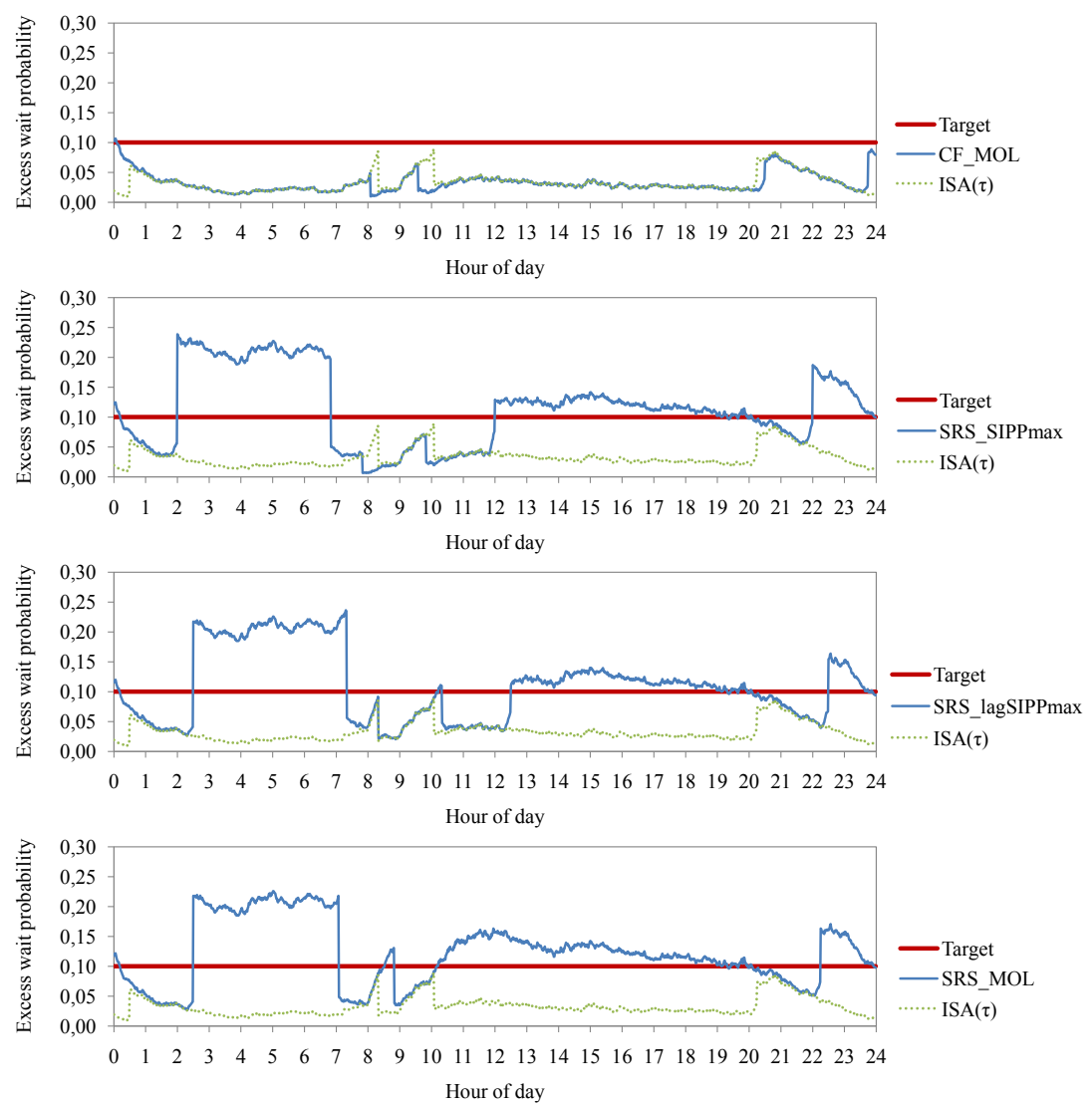

Figure 10: Comparison ISA $(\tau)$ staffing vs. other heuristics: Performance (small system, exponential service and abandonment times)

form formula results in slightly better staffing vectors, although again, it has the tendency to overstaff as the presence of abandonments is ignored (for this example, the overstaffing remains limited due to the low abandonment rate).

Figures 11 and 12 reveal a key advantage of $\operatorname{ISA}(\tau)$. Whereas in a Markovian setting, the SRS_MOL heuristic yielded performance comparable to $\operatorname{ISA}(\tau)$ for the large system, this no longer holds when general service and abandonment times prevail: the excess wait probability is considerably above target. 
For the remaining heuristics, the insights from the Markovian setting largely remain valid: i.e., performance is poor. Consequently, we may conclude that $\operatorname{ISA}(\tau)$ is the only heuristic that yields consistent and satisfactory performance, both for small and large systems, and in particular in settings where the Markovian assumptions do not hold.

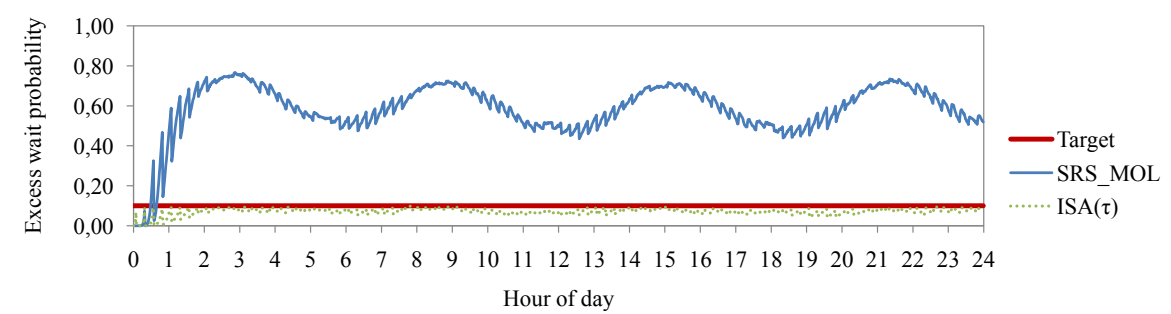

Figure 11: Comparison ISA $(\tau)$ staffing vs. other heuristics: Performance (large system, lognormal service and abandonment times, SCV $=0.5$ )

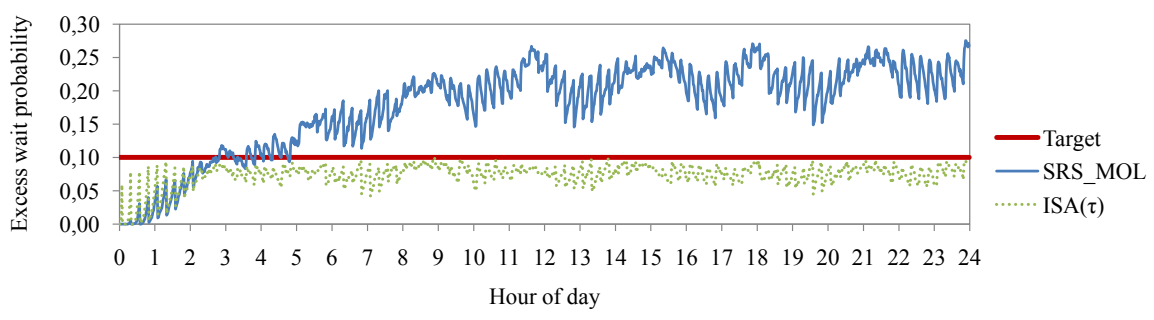

Figure 12: Comparison ISA $(\tau)$ staffing vs. other heuristics: Performance (large system, lognormal service and abandonment times, $\mathrm{SCV}=1.5$ )

\section{Conclusions and future research}

This paper suggests an extension of the simulation-based Iterative Staffing Algorithm (ISA) proposed by Feldman et al. [7] as a method to set staffing levels in small-scale systems with time-varying demand. This extension (called ISA $(\tau)$ ) enables to measure performance based on the probability of 
an excessive wait, instead of the common focus on delay probability as a performance metric. Moreover, it takes into account the sensitivity of small scale systems to changes in the staffing levels, and the presence of staffing intervals. Meanwhile, the advantages of the traditional ISA (namely general applicability, automatic validation) remain valid.

Experiments illustrate that $\operatorname{ISA}(\tau)$ is both effective and efficient in determining staffing requirements for small-scale and large-scale systems, and consistently outperforms heuristics based on stationary approximations, in particular for settings in which the service and abandonment processes are not Markovian. In general, the efficiency of the algorithm tends to depend on its parameters (the amplification factor in Expression 7 and the SCVbased stop criterion can be tuned), and the size of the system (larger systems require more computation time).

Given that $\operatorname{ISA}(\tau)$ is both effective and efficient in detecting required capacities, and requires no specific tools other than simulation, we are confident that the method offers opportunities to support decisions in practice. Though this paper has focused on the context of staffing in an emergency department, the methodology is in fact applicable to any setting in which demand is time-varying, and in which the decision maker relies primarily on capacity to ensure adequate customer service. In future research, we plan to apply the algorithm to a real-life ED setting including patients of different priority classes (due to the lack of detailed process data, the current ED test setting only mimicked real-life arrival data). This requires the inclusion of a triage (or "sorting") step, which may result in a reduction of work at the treatment step. As such, it may impact the service rate of the treatment step, and opens up the opportunity to use different waiting time targets for different priority classes ([5]). In addition, we plan to introduce shift 
constraints in the model, to further increase its practical relevance.

\section{Acknowledgments}

This research was supported by the Research Foundation-Flanders (FWO) (grant no G.0547.09).

\section{Appendix A: Impact of scaling factor on algorithm convergence}

\begin{tabular}{l|cccc|cccc}
\hline & \multicolumn{5}{|c|}{ Small system } & \multicolumn{5}{c}{ Large system } \\
& $i / 2$ & $i$ & $2 i$ & $i^{2}$ & $i / 2$ & $i$ & $2 i$ & $i^{2}$ \\
\hline $\begin{array}{l}\text { Number } \\
\text { iterations }\end{array}$ & 2 & 6 & 5 & 7 & 17 & 17 & 18 & 79 \\
$\begin{array}{l}\text { PHASE I } \\
\text { Number }\end{array}$ & & & & & & & & \\
iterations & 3 & 5 & 5 & 5 & 10 & 6 & 7 & 10 \\
PHASE II & & & & & & & & \\
$\max _{t}\{P(t)\}$ & 0.090 & 0.090 & 0.090 & 0.086 & 0.100 & 0.100 & 0.099 & 0.100 \\
Staffing cost & 74 & 74 & 74 & 74 & 2299.75 & 2257.25 & 2257.75 & 2258 \\
\hline
\end{tabular}

\section{References}

[1] F. Aguado-Correa, N. Padilla-Garrido, M.I. Rengel-Domnguez, M.T. LealLinares. The assumption of exponential interarrival times in hospital emergency departments: right or wrong? Proceedings of the 18th International Annual EurOMA Conference, 3 - 6 July 2011, Cambridge, UK.

[2] R.W. Day, M.D. Dean, R. Garfinkel, S. Thompson, Improving patient flow in a hospital through dynamic allocation of cardiac diagnostic testing time slots, Decision Support Systems 49(4) (2010) 463-473.

[3] M. Defraeye, I. Van Nieuwenhuyse, Controlling Excessive Waiting Times in Emergency Departments: an Extension of the ISA Algorithm, FBE Research Report KBI_1115 (2011) KU Leuven, Belgium. 
[4] M. Defraeye, I. Van Nieuwenhuyse, Setting staffing levels in systems with timevarying demand: the context of an emergency department. FBE Research Report KBI_1102 (2011) KU Leuven, Belgium.

[5] G. Dobson, A. Sainathan, On the impact of analyzing customer information and prioritizing in a service system, Decision Support Systems 51(4) (2011) 875-883.

[6] S.G. Eick,W.A. Massey, W. Whitt, 1993, The Physics of the $M_{t} / G / \infty$ Queue, Operations Research 41(4) (1993) 731-742.

[7] Z. Feldman, A. Mandelbaum, W.A. Massey, W. Whitt, Staffing of TimeVarying Queues to Achieve Time-stable Performance, Management Science $54(2)$ (2008) 324-338.

[8] N. Gans, G. Koole, A. Mandelbaum, Telephone Call Centers: Tutorial, Review, and Research Prospects, Manufacturing \& Service Operations Management 5(2) (2003) 79-141.

[9] O. Garnett, A. Mandelbaum, M. Reiman, Designing a Call Center with Impatient Customers, Manufacturing \& Service Operations Management 4(3) (2002) 208-227.

[10] L.V. Green, P.J. Kolesar, The Pointwise Stationary Approximation for Queues with Nonstationary Arrivals, Management Science 37(1) (1991) 84-97.

[11] L.V. Green, P.J. Kolesar, On the Accuracy of the Simple Peak Hour Approximation for Markovian Queues, Management Science 41(8) (1995) 1353-1370.

[12] L.V. Green, P.J. Kolesar, J. Soares, Improving the SIPP Approach for Staffing Service Systems That Have Cyclic Demands, Operations Research 49(4) (2001) $549-564$. 
[13] L.V. Green, P.J. Kolesar, J. Soares, An improved heuristic for staffing telephone call centers with limited operating hours, Production and Operations Management 12 (2003) 46-61.

[14] L.V. Green, J. Soares, J.F. Giglio, R.A. Green, Using Queueing Theory to Increase the Effectiveness of Emergency Department Provider Staffing, Academic Emergency Medicine 13(1) (2006) 61-68.

[15] L.V. Green, P.J. Kolesar, W. Whitt, Coping with Time-Varying Demand when Setting Staffing Requirements for a Service System, Production and Operations Management 16(1) (2007) 13-39.

[16] D. Gross, J.F. Shortle, J.M. Thompson, C.M. Harris, Fundamentals of Queueing Theory (4th Edition), Wiley Series in Probability and Statistics, WileyBlackwell, 2008.

[17] S. Halfin, W. Whitt, Heavy-Traffic Limits for Queues with Many Exponential Servers, Operations Research 29(3) (1981) 567-588.

[18] R. Hall, D. Belson, P. Murali, M. Dessouky, Modeling Patient Flows Through the Healthcare System, in: R. W. Hall, (Ed.), Patient Flow: Reducing Delay in Healthcare Delivery, Springer US, 2006, 91, pp. 1-44.

[19] A. Ingolfsson, E. Akhmetshina, S. Budge, L. Yongyue, W. Xudong, A Survey and Experimental Comparison of Service-Level-Approximation Methods for Nonstationary M(t)/M/s(t) Queueing Systems with Exhaustive Discipline, INFORMS Journal on Computing 19 (2007) 201-214.

[20] F. Iravani, B. Balcioglu, Approximations for the M/GI/N+GI type call center, Queueing Systems 58 (2008) 137-153.

[21] D.L. Jagerman, Nonstationary Blocking in Telephone Traffic, Bell System Technical Journal 54(3) (1975) 625-661. 
[22] O.B. Jennings, A. Mandelbaum, W.A. Massey, W. Whitt, Server Staffing to Meet Time-Varying Demand. Management Science 42(10) (1996) 1383-1394.

[23] G. Koole, A. Mandelbaum, Queueing Models of Call Centers: An Introduction, Annals of Operations Research 113(1-4) (2002) 41-59.

[24] A. Mandelbaum, S. Zeltyn, Service Engineering in Action: The Palm/ErlangA Queue, with Applications to Call Centers, in: D. Spath, K.-P. Fähnrich, (Eds.), Advances in Services Innovations, Springer Berlin Heidelberg, 2007, pp. $17-45$.

[25] W.A. Massey, W. Whitt, An Analysis of the Modified Offered-Load Approximation for the Nonstationary Erlang Loss Model, The Annals of applied probability 4(4) (1994) 1145-1160.

[26] W.A. Massey, W. Whitt, Peak Congestion in Multi-Server Service Systems with Slowly Varying Arrival Rates, Queueing Systems 25(1-4) (1997) 157-172.

[27] M.L. McCarthy, S.L. Zeger, R. Ding, D. Aronsky, N.R. Hoot, G.D. Kelen, The Challenge of Predicting Demand for Emergency Department Services, Academic Emergency Medicine 15(4) (2008) 337-346.

[28] P. Walley, Designing the accident and emergency system: lessons from manufacturing, Emergency Medicine Journal 20 (2003) 126-130.

[29] W. Whitt, The pointwise stationary approximation for $\mathrm{M}_{t} / \mathrm{M}_{t} / \mathrm{s}$ Queues is Asymptotically Correct As the Rates Increase, Management Science 37(3) (1991) 307-314.

[30] W. Whitt, Understanding the Efficiency of Multiserver Service Systems, Management Science 38(5) (1992) 708-723.

[31] W. Whitt, Engineering solution of a basic call-center model, Management Science 51(2) (2005) 221-235. 
[32] W. Whitt, What You Should Know About Queueing Models to Set Staffing Requirements in Service Systems, Naval Research Logistics 54(5) (2007) 476484 .

[33] S. Zeltyn, A. Mandelbaum, Call centers with impatient customers: Manyserver asymptotics of the $M / M / n+G$ queue, Queueing Systems 51(3-4) (2005) 361-402.

Mieke Defraeye is a Ph.D. candidate at the Research Center for Operations Management at KU Leuven, Belgium. She received her M.Sc. degree in Business Engineering at KU Leuven in 2009. Her research interests include capacity planning and scheduling under uncertainty, and healthcare operations management.

Inneke Van Nieuwenhuyse is Associate Professor at the Research Center for Operations Management, KU Leuven, Belgium. Her research interests include operations management, more specifically the design and analysis of stochastic manufacturing and service systems. She has been involved in applied research projects for industrial and consulting companies as well as hospitals. Her work has been published in a.o. European Journal of Operational Research, IIE Transactions, and International Journal of Production Economics. 